COMPUTATIONAL METHODS IN APPLIED MATHEMATICS, Vol.7(2007), No. 2, pp.118-134

(C) 2007 Institute of Mathematics of the National Academy of Sciences of Belarus

\title{
ON PARALLEL NUMERICAL ALGORITHMS FOR SIMULATING INDUSTRIAL FILTRATION PROBLEMS
}

\author{
R. ČIEGIS ${ }^{1}$, O. ILIEV ${ }^{2}$, AND Z. LAKDAWALA ${ }^{2}$
}

\begin{abstract}
The performance of oil filters used in the automotive industry can be significantly improved, especially when computer simulation is an essential component of the design process. In this paper, we consider parallel numerical algorithms for solving mathematical models describing the process of filtration, filtering solid particles out of liquid oil. The Navier - Stokes - Brinkmann system of equations is used to describe the laminar flow of incompressible isothermal oil. The space discretization in the complicated filter geometry is based on the finite-volume method. Special care is taken for an accurate approximation of the velocity and pressure on the interface between the fluid and the porous media. The time discretization used here is a proper modification of the fractional time step discretization (cf. Chorin scheme) of the NavierStokes equations, where the Brinkmann term is considered in both the prediction and the correction substeps.

A data decomposition method is used to develop a parallel algorithm, where the domain is distributed among the processors by using a structured reference grid. The MPI library is used to implement the data communication part of the algorithm. A theoretical model is proposed for the estimation of the complexity of the given parallel algorithm and a scalability analysis is done on the basis of this model. The results of the computational experiments are presented, and the accuracy and efficiency of the parallel algorithm is tested on real industrial geometries.
\end{abstract}

2000 Mathematics Subject Classification: 65N20; 65M25.

Keywords: parallel algorithms, numerical algorithms, mathematical modelling, filtration processes.

\section{Introduction}

The numerical solution of nonlinear PDEs, such as porous media models or Navier-Stokes equations, require much computational effort. A significant step in reducing the CPU time and/or increasing the accuracy of the simulations is the usage of parallel computers and clusters of workstations. The power of modern personal computers is increasing steadily, but not enough to satisfy all scientific and engineering computational demands. In such cases, parallel computing may be the answer. Parallel computing not only gives access to increasing computational resources but it also becomes economically feasible. This is mainly because clusters of workstations can be used as local dedicated computational nodes or as a parallel computer, according to momental needs of a department. A good review on the state of the art in numerical solution of PDEs on parallel computers is given in [7]. This book surveys

\footnotetext{
${ }^{1}$ Vilnius Gediminas Technical University, Sauletekio al. 11, LT-10223, Vilnius, Lithuania. E-mail: rc@fm.vtu.lt

${ }^{2}$ Fraunhofer ITWM, Fraunhofer-Platz 1, D-67663 Kaiserslautern, Germany. E-mail: \{iliev, lakdawala\} Qitwm.fhg.de
} 
the major topics that are essential to high-performance simulation on parallel computers, including programming models, load balancing, mesh generation, efficient numerical solvers, and scientific software.

Filtering solid particles out of liquid oil is very essential for automotive engines (as well as for many other applications). An oil filter can be described shortly as a filter box (which could be of complicated shape) with an inlet(s) for dirty oil and an outlet(s) for filtered oil. The inlet(s) and outlet(s) are separated by a filtering medium, which is usually a single-layer or a multilayer porous media. The optimal design for the filter housing, achieving optimal pressure drop - flow rate ratio, etc., requires thorough knowledge about the field of flow through the filter. Accurate information about the velocity and pressure distributions can be obtained from a 3-D computer simulation of the fully coupled flow: the flow in the area between the inlet(s) and the filtering medium, the flow within the filtering medium, and the flow between the filtering medium and the outlet(s). The general-purpose commercial CFD software might be inefficient and/or inaccurate in computing such particular flows. An efficient numerical algorithm for such problems was developed. It was further implemented to satisfy the needs of the existing oil filter manufacturing company, namely IBS Filtran. Intensive collaboration with the company has made it possible to develop specialized software, namely SuFiS (Suction Filter Simulation). The single grid version of the algorithm is shortly presented in $[19,20]$ (for more detail, see [27]).

The top and bottom pans of a typical filter housing are shown in Fig. 1.1, and the crosssection of the filter assembly is schematically represented in Fig. 1.2. Several challenging problems have to be solved to support the design of oil filters: detailed simulation of coupled
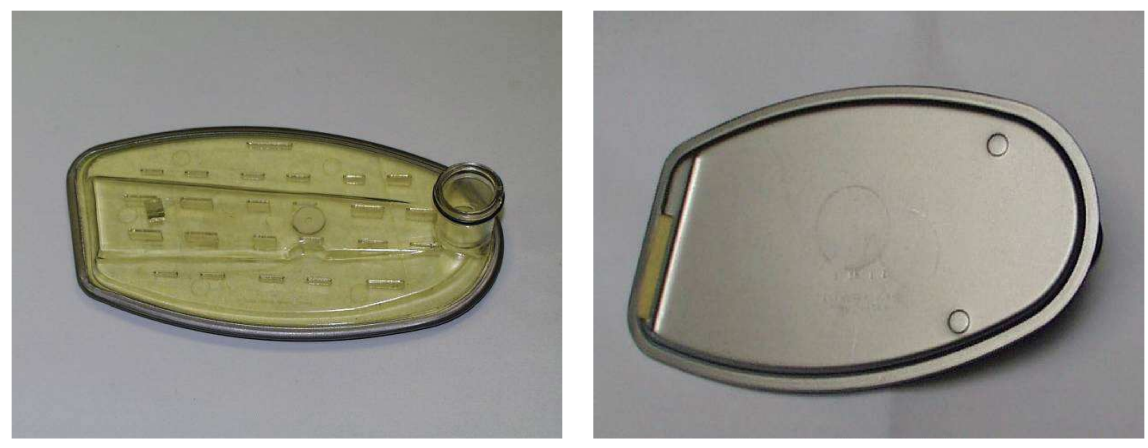

Fig. 1.1. Top pan (left) and bottom pan (right) of the filter housing

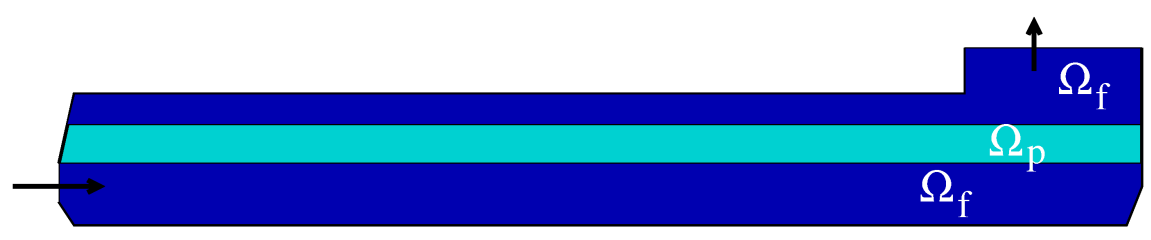

Fig. 1.2. Schematic representation of the vertical cross-section of the filter

flows through filters; modeling and simulation of the capturing of impurity particles by the filtering media; interaction between the flow and the deformable filtering medium; optimal design, and so on. Here we discuss the first of these problems, i.e., the coupled flow simulations. More precisely, we discuss the development of efficient numerical algorithms and software for the simulation of a 3-D flow through oil filters. We also give special attention to the use of correct parameters and to the validation of the software. Note that some details 
concerning modelling and simulation of the capturing of particles by the filtering medium, as well as of related filtration processes, can be found in [16].

This paper aims to discuss the parallelization of the existing industrial sequential code $\mathrm{SuFiS}$, which is extensively used for the simulation of fluid flows in industrial filters. The domain or data parallelization paradigm is used to build a parallel algorithm [25]. The MPI library is used to implement the data communication among processors.

The paper is organized as follows. In Section 2, we first formulate the problem. The following two subsections describe the fractional time step discretization and the Finite Volume $(\mathrm{FV})$ discretization method used for solving the flow problem, respectively. A parallel algorithm is described in Section 3, which is based on the parallel domain (data) decomposition method. The systems of linear equations are solved by a parallel version of the preconditioned BiConjugate Gradient Stabilized algorithm. A theoretical model evaluating the complexity of the parallel SuFiS algorithm is proposed. The results of computational experiments corresponding to the SP5 computer and a cluster of workstations are presented and the efficiency of some popular parallel preconditioners is investigated. During computations, the diagonal and the incomplete LU (ILU) factorization preconditioners are considered. A parallel version of ILU is obtained by doing factorization of a local part of the matrix on each processor. It is well known that such strategy reduces the convergence rate of the iterative algorithm, however, the parallelism of the obtained preconditioner is the same as that obtained for the diagonal one. In Section 4, the computational results of the experiments are presented, where some industrial filters are simulated and flows through such filters are investigated. Some final conclusions are given in Section 5.

\section{Governing equations and space and time discretization}

2.1. Governing equations. The Brinkmann model (see, e.g., $[6,24]$ ) describing the flow in porous media, $\Omega_{p}$, and the Navier-Stokes equations (see, e.g., [13]) describing the flow in the pure fluid region, $\Omega_{f}$, together with the interface conditions for the continuity of the velocity and the continuity of the normal component of the stress tensor, are reformulated so that a single system of partial differential equations governs the flow in the pure liquid and in the porous media. This is done using the fictitious region method. Note that the fictitious region method permits using one system of equations to treat the fluid and the porous and solid regions simultaneously (see, e.g., $[1,30,31]$ ). The coefficients of the equations vary so that the single system is reduced to the Navier - Stokes equations in the liquid zone and to the Brinkmann-like model in the porous media. This approach is relevant when the interface conditions between the plain (fluid) and porous media are chosen to be the continuity of the velocity and the continuity of the normal component of the stress tensor. For theoretical justification of this approach, for the case of the Stokes flow, see $[1,30]$ and the references therein.

The Navier — Stokes - Brinkmann system of equations describing a laminar, incompressible and isothermal flow throught the domain reads

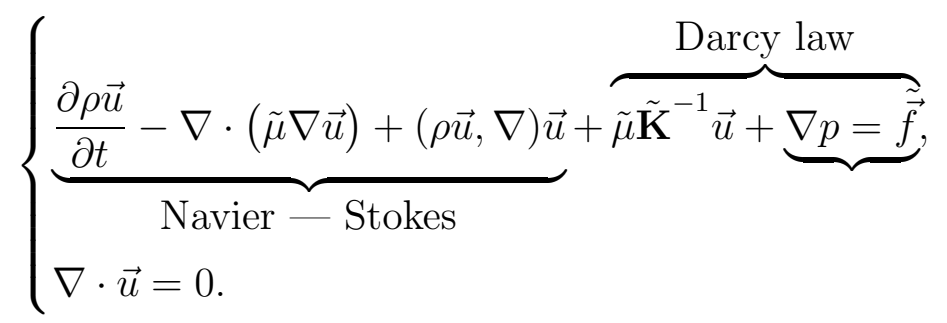


In this case, the tilde-quantities are defined as follows:

$$
\tilde{\mu}=\left\{\begin{array}{ll}
\mu & \text { in } \Omega_{f}, \\
\mu_{\text {eff }} & \text { in } \Omega_{p},
\end{array} \quad \tilde{\vec{f}}=\left\{\begin{array}{ll}
\vec{f}_{N S} & \text { in } \Omega_{f}, \\
\vec{f}_{B} & \text { in } \Omega_{p},
\end{array} \quad \tilde{\mathbf{K}}^{-1}= \begin{cases}0 & \text { in } \Omega_{f}, \\
\mathbf{K}^{-1} & \text { in } \Omega_{p} .\end{cases}\right.\right.
$$

Here $\vec{u}$ and $p$ stand for the velocity vector and the pressure, respectively, and $\rho, \mu, \mathbf{K}$ denote the density, the viscosity, and the permeability tensor of the porous medium, respectively.

No slip boundary condition is a boundary condition which requires that the fluid in container's walls be at rest.

2.2. Time discretization. The choice of the time discretization influences the accuracy of the numerical solution and the stability of the algorithm (e.g., the restrictions on the time step). In this paper, we are interested in the steady state solution only, when the steady state solution is a limit of the unsteady one for $t \rightarrow \infty$. Thus stability and fast convergence are the criteria influencing the choice of the time discretization here. Unsteady regimes will be the subject of another paper.

Before the time discretizations are presented, some designations are introduced. The operators corresponding to the discretized convective and diffusive terms in the momentum equations are denoted by $C(\vec{u}) \vec{u}$ and $D \vec{u}$, respectively. The particular form of these operators depends on the space discretization and will be discussed below. Further, the discretization of the gradient is denoted by $G$, and $G^{T}$ denote the discretization of the divergence operator. Finally, B $\vec{u}$ denoted the operator corresponding to the Darcy term, namely $\mu \tilde{\mathbf{K}}^{-1} \vec{u}$, in the momentum equations. In some cases, the same notation is used for the continuous and discrete functions if this does not cause misunderstanding. Below, the superscript ${ }^{n}$ is used to denote the values at the old time level, and ${ }^{n+1}$ or $\mathrm{NO}$ - the values at the new time level. Theotation $\tau$ stands for the time step, $\tau=t^{n+1}-t^{n}$. With these notations, the following fractional time step discretization scheme can be written:

$$
\begin{gathered}
\left(\rho \vec{u}^{n+1 / 2}-\rho \vec{u}^{n}\right)+\tau\left(C\left(\vec{u}^{n}\right)-D+B\right) \vec{u}^{n+1 / 2}=\tau G p^{n}, \\
\left(\rho \vec{u}^{n+1}-\rho \vec{u}^{n+1 / 2}\right)+\tau\left(B \vec{u}^{n+1}-B \vec{u}^{n+1 / 2}\right)=\tau\left(G p^{n+1}-G p^{n}\right), \quad G^{T} \rho \vec{u}^{n+1}=0 .
\end{gathered}
$$

This algorithm for the Navier - Stokes - Brinkmann equations can be viewed as a modification of the well-known Chorin method for Navier - Stokes equations. The sum of the first and the second equations above gives an implicit discretization of the momentum equations. The first equation is solved with respect to the velocities, using the old value of the pressure gradient, thus obtaining a prediction for the velocity. To solve the second equation for pressure correction, one takes the divergence from it and uses the continuity equation. The result is a Poisson-type equation for the pressure correction. There exists extensive mathematical literature on first- and second- order fractional time step discretizations, incremental and non-incremental forms of equations, stability, splitting of the boundary conditions, etc. Some discussions on this topic, as well as further references, can be found in $[8,14]$.

As mentioned above, the Darcy term in the Navier - Stokes - Brinkmann equations needs special treatment. In equation (2.2), this term is taken into account at both time substeps. Note that the pressure correction equation should be carefully derived in this case. A naive application of the Chorin method would give

$$
G^{T}\left(\rho \vec{u}^{n+1}-\rho \vec{u}^{n+1 / 2}\right)+G^{T} \tau\left(B \vec{u}^{n+1}-B \vec{u}^{n+1 / 2}\right)=G^{T} \tau\left(G p^{n+1}-G p^{n}\right) .
$$


Denoting $q$ for the pressure correction, where $q=p^{n+1}-p^{n}$, one can rewrite the above equation as

$$
G^{T} \tau G q=G^{T}\left(\rho \vec{u}^{n+1}-\rho \vec{u}^{n+1 / 2}\right)+G^{T} \tau\left(B \vec{u}^{n+1}-B \vec{u}^{n+1 / 2}\right) .
$$

Furthermore, using the continuity equation $G^{T} \rho u^{n+1}=0$, and assuming that $G^{T} B u^{n+1} \approx 0$, the above equation reduces to

$$
G^{T} \tau G q=-G^{T}\left(\rho \vec{u}^{n+1 / 2}+\tau B \vec{u}^{n+1 / 2}\right) .
$$

A drawback of this pressure correction equation is that its operator does not "see" the porous media, and that the continuity equation is approximately satisfied in the porous media. For a constant time step, its operator is equivalent to the Poisson equation with constant coefficients.

In order to achieve a better convergence, let us consider another approach for forming a pressure correction equation. Denote $I$ as the identity $3 \times 3$ matrix. Rewriting equation (2.2) gives

$$
\left(I+\frac{\tau}{\rho} B\right) \rho \vec{u}^{n+1}-\left(I+\frac{\tau}{\rho} B\right) \rho \vec{u}^{n+1 / 2}=\tau\left(G p^{n+1}-G p^{n}\right) .
$$

Keeping in mind that $\left(I+\frac{\tau}{\rho} B\right)^{-1}$ always exists because $B$ is positive definite, the above equation can be transformed to

$$
\left(\rho \vec{u}^{n+1}-\rho \vec{u}^{n+1 / 2}\right)=\left(I+\frac{\tau}{\rho} B\right)^{-1} \tau\left(G p^{n+1}-G p^{n}\right) .
$$

Now, applying the divergence operator, $G^{T}$, to this equation, and using the continuity equation, the following pressure correction equation is obtained:

$$
G^{T}\left(I+\frac{\tau}{\rho} B\right)^{-1} \tau G q=-G^{T} \rho \vec{u}^{n+1 / 2} .
$$

It is easy to see that the derived pressure correction equation in the pure fluid region, where $B=0$, reduces to the standard Chorin scheme. The same equation for pure fluids is obtained within the SIMPLEC approach (see [13]), or within the Schur complement approach (see [32]). It is more important to see that this equation has the form of the well-known Darcy equation in the porous medium (note that in the porous medium $I \ll B$ ). Thus, the equation describes both the pure fluid zones and the porous zones equally well.

After the pressure correction equation is solved, the pressure is updated, $p^{n+1}=p^{n}+q$, and the new velocity is calculated based on equation (2.7):

$$
\rho \vec{u}^{n+1}=\rho \vec{u}^{n+1 / 2}+\left(I+\frac{\tau}{\rho} B\right)^{-1} \tau G q .
$$

2.3. Grid and finite volume discretization in space. The geometrical information about the computational domain is usually provided in a CAD format, for example, the stl format. A pre-processor based on the Level Set Method (see [15]), is used to process the given CAD data for attaining the assembly of a filter housing. The output of the preprocessor is a computational domain (i.e., the internal volume of the filter housing), along with a generated grid. An example of a computational domain is given in Fig. 2.3. 
The governing equations are discretized by the Finite Volume Method (see [13]) on the generated Cartesian grid. A cell-centered grid with a collocated arrangement of the velocity and pressure is used. The Rhie - Chow interpolation (see [12]) is used to avoid spiral oscillations that can appear due to the collocated arrangement of the unknowns. Upwind, central differencing and deferred correction schemes can be used for the convective term. In general, the discretization of convective and the diffusive (viscous) terms in the pure fluid region is close or identical to that described in $[12,13]$, and therefore it will not be described here (some details can be found in our earlier papers, for example, [20-22]. Particular
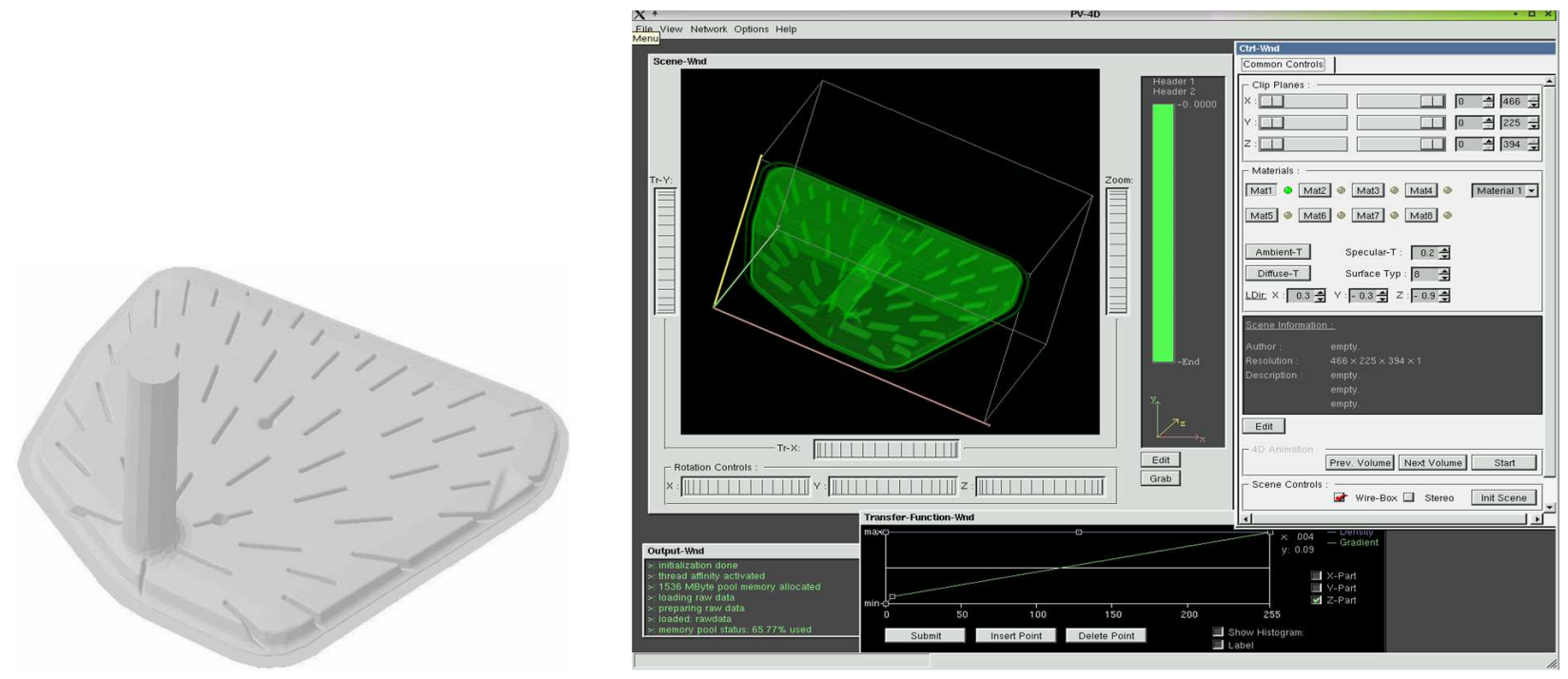

Fig. 2.3. Computational domain of the filter housing (left) and a snapshot of the visualization tool (right)

attention is given to the discretizations near the interfaces between the fluid and the porous medium. Conservativity of the discretization is achieved here by choosing a finite volume method as a discretization technique. To get an accurate approximation for the velocity and pressure on the interface, a special modification of the discretization near the interface is used. First of all, it should be noted that the pressure gradient in the momentum equation is discretized in each cell separately. To do this, the pressure values from the cell centers are interpolated to the cell faces. In the pure fluid region, this is done by linear interpolation where the pressure gradient is discretized via central differences. The most crucial part is the interpolation of pressure on the interface between the pure fluid and the porous regions. The problem-dependent interpolation is employed, which uses the operator from (2.8) together with the mass conservation assumption (i.e., continuity of the normal component of the flux across the interface). For a detailed illustration of this approach, suppose that $p_{L}$ is a pressure value in a pure fluid cell located on the left of the interface, and $p_{R}$ is a pressure value in a porous cell located on the right of the interface. The pressure at the interface is assumed to be continuous, and the value there, $p_{e}$, in the case of isotropic media (i.e., scalar permeability) is calculated from (2.8) as follows:

$$
p_{e}=\frac{\left(1+\frac{\tau}{\rho} b\right)_{L}^{-1} p_{L}+\left(1+\frac{\tau}{\rho} b\right)_{R}^{-1} p_{R}}{\left(1+\frac{\tau}{\rho} b\right)_{L}^{-1}+\left(1+\frac{\tau}{\rho} b\right)_{R}^{-1}}
$$

where $b$ is the component of $B$ in the isotropic case. 
2.4. Series of computations with different velocities and viscosities. In practice, a series of computations with different velocities and different viscosities have to be performed for a fixed geometry. Particularly, in the case of oil filter simulations, the performance of a filter is usually evaluated for different flow rates and different temperatures, i.e. for different viscosities and different densities, since they depend on temperature. Also, recall that each single computation is performed for an incompressible nonisothermal fluid. In order to reduce computational efforts, we use a special technique to define the initial approximation. This routine takes advantage of the fact that there exists a good initial guess for all simulated cases except for the first one. The cases to be simulated are ordered so that the parameter

$$
\gamma_{l}=\frac{1}{\mu_{l} Q_{l}}
$$

is increasing. Here $Q$ stands for the prescribed flow rate at the inlet, and $l$ stands for the current number of input parameters. As soon as the first case corresponding to $\gamma_{l}$ is computed, computations corresponding to $\gamma_{l+1}$ start with reading the $l$ th steady-state solution, and rescaling thereby the pressure in accordance with the formula

$$
G p_{l+1}=\frac{\gamma_{l}}{\gamma_{l+1}} G p_{l} \text {. }
$$

2.5. Implementational sketch. At the end of this section we summarize the proposed discrete scheme and numerical algorithms. Figure 2.4 gives a short description of the sequential SUFIS algorithm.

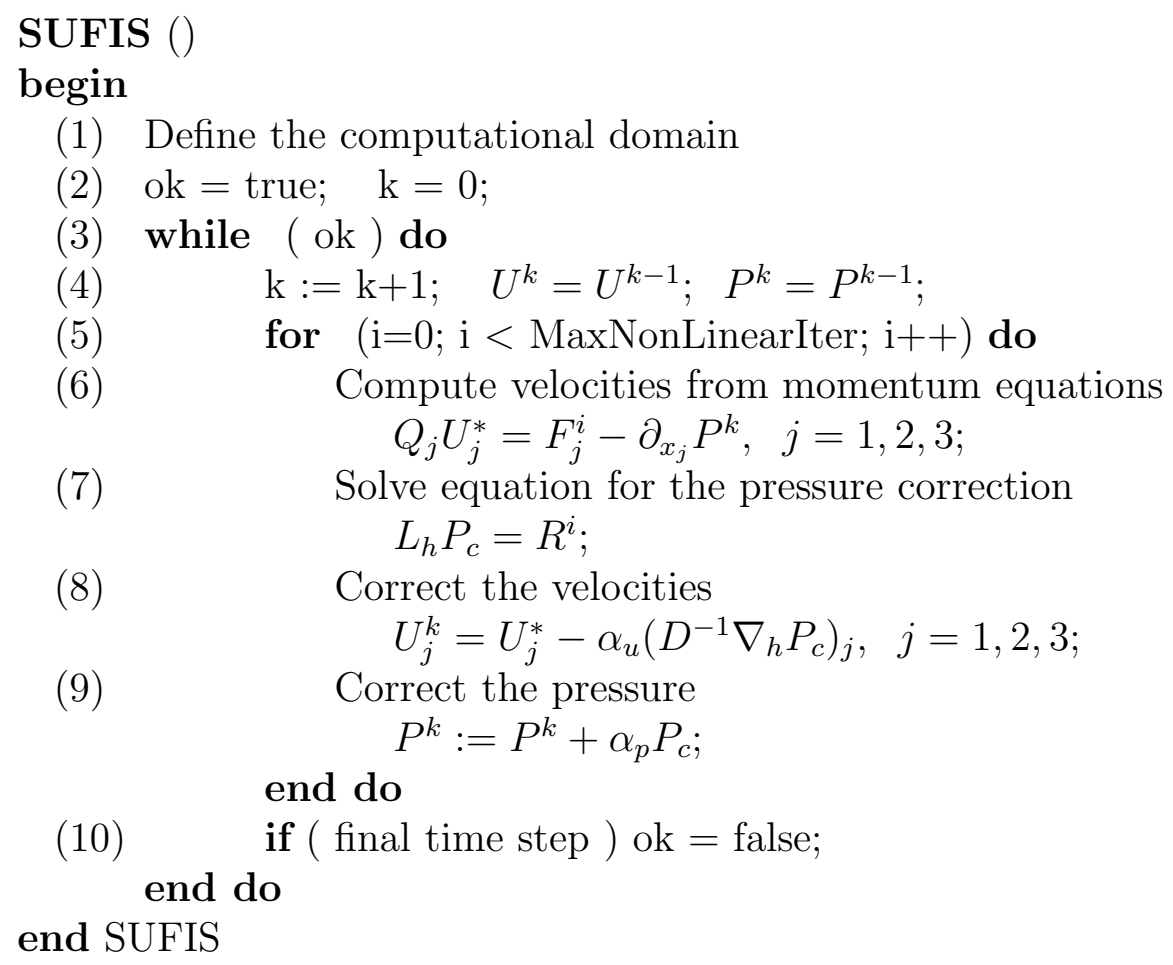

Fig. 2.4. Sequential SUFIS algorithm

In each iteration at steps (6) and (7) four systems of linear equations are solved. For many problems this part of the algorithm requires up to $90 \%$ of the total CPU time. We use the BiCGSTAB algorithm that solves the asymmetric linear system

$$
A x=f
$$


by using the preconditioned BiConjugate Gradient Stabilized method [4]. A short description of the sequential BiCGSTAB algorithm is given in Fig. 2.5.

BiCGSTAB (Vec x, Vec f, Matrix A, double $\varepsilon$ )

begin

(1) Compute the precondinioner $D$;

(2) normf $=\|f\|$;

(3) if $\quad($ normf $<\varepsilon)$ normf $=1$;

(4) $r=f-A x$;

(5) $\tilde{r}=r ; \quad$ ok $=$ true; $\quad \mathrm{i}=0$;

(6) while (ok) do

(7) $\quad \mathrm{i}++; \quad \rho_{1}=(\tilde{r}, r)$;

(8) if $(\mathrm{i}==1)$ then

(9) $\mathrm{p}=\mathrm{r}$;

else

$$
\begin{aligned}
& \beta=\left(\rho_{1} \alpha\right) /\left(\rho_{2} \omega\right) ; \\
& p=r+\beta(p-\omega v) ; \\
& \text { end if }
\end{aligned}
$$$$
D \hat{p}=p ;
$$$$
v=A \hat{p}
$$$$
\alpha=\rho_{1} /(\tilde{r}, v) \text {; }
$$$$
s=r-\alpha v \text {; }
$$$$
\text { if }(\|s\| / \text { normf }<\varepsilon) \text { then }
$$$$
x+=\alpha \hat{p} \text {; }
$$$$
\text { ok }=\text { false; }
$$

else

$$
D \hat{s}=s
$$$$
t=A \hat{s} \text {; }
$$$$
\omega=(t, s) /(t, t) \text {; }
$$$$
x+=\alpha \hat{p}+\omega \hat{s} ; \quad r=s-\omega t ;
$$$$
\rho_{2}=\rho_{1} \text {; }
$$$$
\text { if }(\|r\| / \text { normf }<\varepsilon) \text { ok }=\text { false; }
$$

end if

end do

end BiCGSTAB

Fig. 2.5. Serial BiCGSTAB algorithm

\section{Parallel algorithm}

In this section, the essential details of the developed parallel algorithm are described. A theoretical model estimating the complexity of the parallel SuFiS algorithm is also proposed.

As stated above, the solver for the linear system of equations typically requires up to $70-90$ percent of the total computation time. Thus, there is a possibility to parallelize only the linear system solver by using one of the well-known parallel software packages such as PETSc [3]. Alternatively, in many cases, the main goal of parallel computations is not only to increase the size of simulated problems but also to solve them faster. Therefore, the 
distribution of the discretization part of the algorithm to scale the problem size according to the increased number of processors becomes a crucial point to consider. Also, when only the linear solver is parallelized, computing the discretization is done only on the master processor using the sequential code, while the linear systems of equations are solved in parallel. The drawback of this practice becomes apparent when the additional costs, pertaining to the distribution of the matrix and the right-hand side vector among processors and assembling the solution on the master processor are considered.

3.1. Domain decomposition. The Navier-Stokes-Brinkmann system of equations (2.1) is solved in a complicated 3D region. A discrete grid is described as a general nonstructured set of finite-volumes. The discretization of the PDEs and assembling of the coefficients of the linear equations is done by using a general technique, which is standard for the solvers of flows in porous media and Navier-Stokes equations $[5,26]$. The goal of the domain (data) decomposition method is to define a suitable mapping of all finitevolumes $V$ onto the set of $p$ processors

$$
V=V_{1} \cup V_{2} \cup \ldots \cup V_{p}
$$

where $V_{j}$ defines the elements mapped onto $j$-th processor. The load balancing problem should be solved during the implementation of this step. First, it is essential that each processor has about the same number of elements, since this number will determine the computational complexity for all parts of the SuFiS algorithm. Due to the stencil of discretization, the computational domains of the processors can overlap. The information belonging to the overlapped regions should be exchanged between the processors. For distributed memory computers, the MPI library is used to send explicit messages between the processors, contributing to the additional costs of the parallel algorithm. Thus, the second goal of defining the optimal data mapping is to minimize the overlapping regions.

The $p$-way graph partitioning problem is $N P$-complete, i.e., no polynomial time algorithm is likely to be found to solve this problem. Therefore, heuristic algorithms have been developed to find a good solution in a reasonable time. The multilevel partitioning method is one of the most efficient partitioning methods having a linear time complexity. The stateof-the-art implementation of a family of multilevel partitioning methods for partitioning unstructured graphs and hypergraphs is available as a METIS software library [23].

A simpler domain decomposition algorithm has been used for this particular case. It takes into account that the orthogonal 3D structured grid is used as a reference grid for the definition of the computational grid. Therefore, standard 3D decomposition of the processors $p_{1} \times p_{2} \times p_{3}$ can be used. Such a strategy simplifies the implementation of the data exchange algorithms, since it is very easy to define the neighbours of each processor and the overlapping elements.

3.2. Load balancing. In order to solve the load balancing problem for a given number of processors, all combinations of 3D processors topologies have been generated. A topology with the best load balancing that minimizes the number overlapping elements has been found.

In a series of computational experiments, the quality of the obtained partitioning were tested and compared to the partitioning computed by the METIS. Table 3.1 presents the values of the load disbalance parameter $d_{p}$ and the number of overlapping elements $w_{p}$ (or edges cutting the partitioned subsets of elements in the case of METIS partitioning) are presented for the METIS and orthogonal 3D partitioning. The grid was generated for a real industrial application, the graph of this grid had 596094 nodes and 1507732 edges, the auxiliary structured grid had 5428000 nodes. 
It is seen that the simple grid partitioning algorithm gives mappings with good load balancing and the number of overlapped elements is also close to the number of similar elements in the partitioning METIS generated.

Table 3.1. Experimental investigation of the quality of partitioning algorithms

\begin{tabular}{|c|c|c|c|c|}
\hline$p$ & $d_{p, \text { Metis }}$ & $2 w_{p, \text { Metis }}$ & $d_{3 D}$ & $w_{3 D}$ \\
\hline 2 & 1.0 & 6090 & 1.05 & 5874 \\
4 & 1.0 & 12164 & 1.19 & 16884 \\
8 & 1.0 & 24162 & 1.21 & 34450 \\
12 & 1.0 & 32836 & 1.22 & 60270 \\
\hline
\end{tabular}

Now the costs of the data initialization are estimated. The master processor reads the information on the grid from a file and broadcasts it to the other processors. The complexity of the global broadcast operation strongly depends on the architecture of the parallel computer (see $[10,17,18]$ ). The cost of broadcasting $n$ data items of between $p$ processors is estimated as

$$
B(n, p)=R(p)\left(\alpha_{b}+\beta_{b} n\right),
$$

where $R(p)$ depends on the algorithm used to implement the broadcast operation and the architecture of the computer. For the simplest algorithm, $R(p)=p$. Taking into account the time $\mathcal{O}(n)$ required to read data from the file and assuming that $\alpha_{b} \ll \beta_{b} n$, a bound on costs of grid initialization is found

$$
W_{p, \text { init }}=\left(c_{0}+\beta_{b} p\right) n \text {. }
$$

Note that this part of computations does not depend on the number of nonlinear iterations and the number of time steps. Therefore, the initialization costs can be neglected for problems where a long transition time is simulated.

3.3. Parallel discretization. The sequential algorithm is decomposed into local computations supplemented with corresponding communication operations. The matrices and the right-hand side vectors are assembled element by element. This can be done locally by each processor, if all ghost values of the vectors belonging to overlapping regions are exchanged between the processors. The data communication is implemented by an odd-even type algorithm and can be done in parallel between different pairs of processors. Thus, we can estimate the costs of the data exchange operation as

$$
W_{\mathrm{exch}}=\alpha_{e}+\beta_{e} m
$$

where $m$ is the number of items sent between two processors, $\alpha$ is the message startup time, and $\beta$ is the time required to send one data element.

The time required to calculate all coefficients of the discrete problem is given by

$$
W_{p, \text { coeff }}=c_{1} d_{p} \frac{n}{p}
$$

where $d_{p}$ is a load disbalance parameter.

3.4. Parallel BiCGSTAB algorithm. The sequential BiCGSTAB algorithm is modified in a way so that its convergence properties are not changed during the parallelization process. The only exception is due to the implementation of the preconditioner $D$. If $D$ is a 
diagonal part of the matrix, i.e., for the Jacobi smoothing a parallel realization of the preconditioner is exactly the same as for the sequential one. In the case of the ILU preconditioner, parallelization can lower the convergence rate of the parallel BiCGSTAB algorithm. These questions will be addressed in the following section. Here, it is assumed that the number of iterations required to solve the systems of linear equations (6), (7) (see the sequential SuFiS algorithm) are the same for the sequential and parallel versions of the BiCGSTAB algorithm.

The following four different operations of the BiCGSTAB algorithm require different data communications between processors.

1. Vector saxpy operations (see steps $(11),(15)$ and (22)) can be computed in parallel if the parameters $\alpha, \beta, \omega$ are given. No communication between the processors is needed, since all required data are locally available on each processor. The complexity of all vector saxpy operations calculated during one iteration is

$$
W_{p, s a x p y}=c_{2} d_{p} \frac{n}{p}
$$

2. Implementation of the matrix-vector multiplication at steps (4), (13) and (20) requires additional information when the boundary nodes of the local part of the vector $x$ are updated (note, that these nodes are inner nodes in the global grid). Such information is obtained by exchanging data with neighbour processors in the specified topology of the processors. The amount of data depends on the grid stencil used to discretize the PDE model, i.e., on the overlap of local subgrids. The communication step can be done in parallel. After exchange of the ghost elements multiplication $A x$ is performed locally on each processor. Taking into account that the matrices are sparse, the complexity of two matrix-vector multiplications during one iteration is estimated by

$$
W_{p, m v}=c_{3} d_{p} \frac{n}{p}+2\left(\alpha_{e}+\beta_{e} m\right) .
$$

3. The computation of the inner products of two vectors at steps (2), (7), (14), (21) and (24) requires a global communication of all processors: first all the processors compute the inner products of the local parts of the vectors, and then these local products are summed up. Different algorithms can be used to implement the global reduction step. In the MPI, there exists a special function MPI_ALLREDUCE, which computes the sum and distributes it to all processors. It is assumed that the MPI library is optimized for each type of supercomputers, taking into account specific details of the computer network. The complexity of computation of all inner products and norms in one iteration is estimated as

$$
W_{p, \text { dot }}=c_{4} d_{p} \frac{n}{p}+5 R(p)\left(\alpha_{r}+\beta_{r}\right) .
$$

For simple implementation of the MPI_ALLREDUCE function, when all processors send their local values to the master processor, which accumulates the results and broadcasts the sum to all processors, $R(p)=c p$.

4. Computation of the preconditioner $D$ is done locally by each processor without any communication operation, the complexity of this step is given by

$$
W_{p, D}=c_{5} d_{p} \frac{n}{p}
$$


The solution of the linear systems $D x=b$ also requires only local computations. Thus, the complexity of steps (12), (19) is given by

$$
W_{p, D^{-1}}=c_{6} d_{p} \frac{n}{p}
$$

After summing up all the estimates, the theoretical model of the complexity of the parallel SUFIS algorithm is achieved:

$$
\begin{gathered}
W_{p}=\left(c_{0}+\beta_{b} p\right) n+K\left(\left(c_{1}+c_{5}\right) d_{p} \frac{n}{p}+c_{7}\left(\alpha_{e}+\beta_{e} m(p)\right)\right)+ \\
+N\left(\left(c_{2}+c_{6}+c_{d o t}\right) d_{p} \frac{n}{p}+c_{8} R(p)\left(\alpha_{r}+\beta_{r}\right)+c_{9}\left(\alpha_{e}+\beta_{e} m(p)\right)\right),
\end{gathered}
$$

where $K$ is the number of steps in the outer loop of the SuFiS algorithm, and $N$ is the total number of BiCGSTAB iterations. Note that the initialization costs (i.e., the first term of the total costs) do not depend on the number of time steps $K$ and they can be neglected in the case where $K$ is a large number.

\section{Results of the computational experiments}

The accuracy of the theoretical complexity model developed above was tested experimentally. Computations were performed on an IBM SP5 computer at CINECA, Bologna and on a Virgo cluster of computers at ITWM, Kaiserslautern. The results of the simulations are presented in Fig. 4.1.
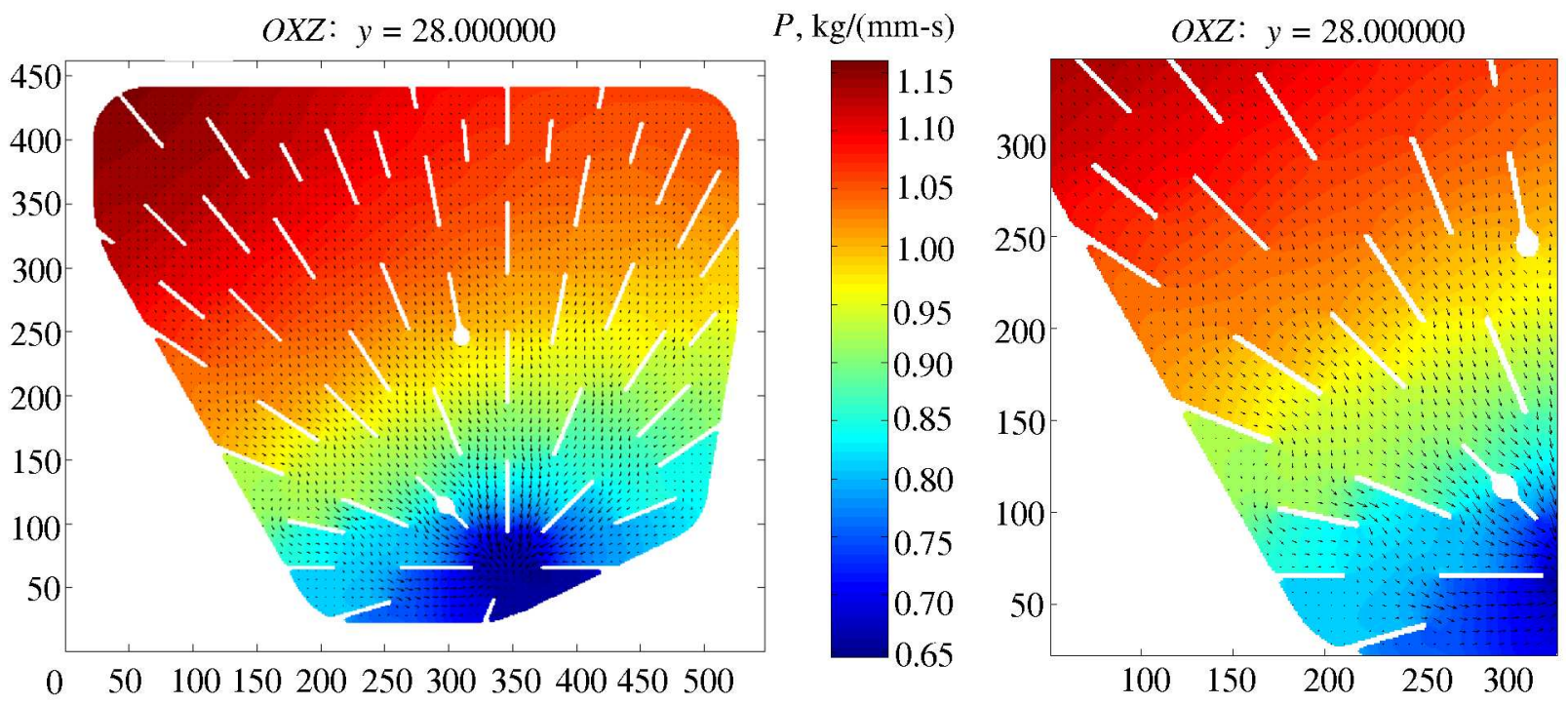

Fig. 4.1. Results of the simulations: velocity (arrows) and pressure (colour) the filter cross-section

The same industrial application as in the example of grid partitioning is used to test the prediction accuracy of the theoretical model.

The following tables present the experimental times and theoretical predictions of the CPU time

$$
T_{\text {init }}=\left(c_{0}+\beta_{b} p\right) n,
$$




$$
\begin{gathered}
T_{1}=K\left(c_{1}+c_{5}\right) d_{p} \frac{n}{p}, \\
T_{2}=N\left(c_{2}+c_{6}\right) d_{p} \frac{n}{p}, \\
T_{\text {exch }}=\left(K c_{7}+N c_{8}\right)\left(\alpha_{e}+\beta_{e} m\right), \\
T_{\text {dot }}=c_{4} d_{p} \frac{n}{p}+c_{8} R(p)\left(\alpha_{r}+\beta_{r}\right) .
\end{gathered}
$$

For each number of processors, the first line gives the experimental values of the CPU and the second lines present the theoretical predictions. Table 4.1 contains the results of the computations for the Virgo cluster of computers.

Table 4.1. Experimental results and theoretical predictions of CPU times for the Virgo cluster

\begin{tabular}{|l|c|c|c|c|c|c|c|c|}
\hline$p$ & $T_{\text {init }}$ & $T_{1}$ & $T_{2}$ & $T_{\text {dot }}$ & $T_{\text {exch }}$ & $T_{\text {total }}$ & $S_{p}$ & $E_{p}$ \\
\hline 1 & 1.66 & 106 & 834 & 117.3 & 0.0 & 1059 & 1 & 1 \\
2 & 3.24 & 54.5 & 435 & 63.0 & 4.7 & 560.5 & 1.89 & 0.95 \\
& 3.24 & 55.7 & 438 & 63.4 & 4.7 & 565.0 & & \\
4 & 4.23 & 31.0 & 252.5 & 36.1 & 6.6 & 330.7 & 3.20 & 0.80 \\
& 4.80 & 31.5 & 248 & 37.0 & 6.1 & 327.7 & & \\
8 & 5.12 & 15.6 & 126.2 & 20.7 & 7.3 & 175.0 & 6.05 & 0.76 \\
& 6.50 & 16.0 & 126.1 & 21.0 & 6.4 & 176.0 & & \\
12 & 7.39 & 10.7 & 84.9 & 15.3 & 5.1 & 123.3 & 8.59 & 0.71 \\
& 9.21 & 10.8 & 84.8 & 15.8 & 7.3 & 126.0 & & \\
\hline
\end{tabular}

The results presented show that the theoretical complexity model gives accurate predictions of different parts of the SuFiS algorithm. The efficiency of the parallel algorithm is also good. We note that the Virgo cluster uses a Myrinet communication network, therefore communication costs do not lower seriously the efficiency of the algorithm for a given number of processors.

The results of the calculations done on the SP5 computer are presented in Table 4.2.

T a ble 4.2. Experimental results and theoretical predictions of CPU times for SP5

\begin{tabular}{|l|c|c|c|c|c|c|c|c|}
\hline$p$ & $T_{\text {init }}$ & $T_{1}$ & $T_{2}$ & $T_{\text {dot }}$ & $T_{\text {exch }}$ & $T_{\text {total }}$ & $S_{p}$ & $E_{p}$ \\
\hline 1 & 1.83 & 82.1 & 735 & 70.3 & 0.0 & 889 & 1 & 1 \\
2 & 3.12 & 44.1 & 381 & 35.7 & 0.58 & 464 & 1.92 & 0.96 \\
& 3.12 & 43.1 & 386 & 37.1 & 0.58 & 470 & & \\
4 & 3.58 & 23.3 & 203 & 19.1 & 3.20 & 252 & 3.52 & 0.88 \\
& 5.16 & 24.4 & 218.7 & 21.4 & 1.2 & 271 & & \\
8 & 4.54 & 11.8 & 93.2 & 10.2 & 3.06 & 122.7 & 7.25 & 0.91 \\
& 6.66 & 12.4 & 111 & 11.6 & 1.9 & 143.6 & & \\
12 & 6.65 & 8.4 & 63.0 & 6.61 & 3.1 & 87.8 & 10.1 & 0.84 \\
& 8.78 & 8.2 & 73.8 & 8.1 & 2.7 & 101.5 & & \\
\hline
\end{tabular}


It can be seen that the theoretical complexity model overestimates the CPU time. The accuracy of the model can be increased by taking into account the well-known fact that the efficiency of vector operations increases. Also, a superlinear speedup of the parallel algorithm is obtained for larger numbers of processors due to the better cache memory utilization in SP5 processors. A simple test was implemented, where the matrix operations $A:=A+B$, $C:=C-D$ were performed many times. The dimensions of the matrices were taken to be $4 \cdot 10^{6}$. The following results were obtained:

$$
\begin{gathered}
T_{1}=35.3, \quad T_{2}=15.3, \quad T_{4}=7.18, \\
T_{8}=2.83, \quad T_{16}=1.29 .
\end{gathered}
$$

4.1. Data distribution by using METIS library. In the previous computations, a 3D data decomposition among the processors was used. Since the geometry of the computational region is rather complicated, such a decomposition leads to an imbalance of the work-load between the processors (up to 1.20 times). Additionally, a general grid distribution algorithm was implemented, which was based on the graph distribution algorithms implemented in the METIS library [23]. Table 4.3 presents the results of the computations

Table 4.3. Experimental results for 3D and METIS data distributions

\begin{tabular}{|l|c|c|c||c|c|c|}
\hline$p$ & $T_{p, 3 D}$ & $S_{p, 3 D}$ & $E_{p, 3 D}$ & $T_{p, M}$ & $S_{p, M}$ & $E_{p, M}$ \\
\hline 1 & 885.7 & 1.00 & 1.00 & 893.0 & 1.00 & 1.00 \\
2 & 480.4 & 1.84 & 0.92 & 459.7 & 1.94 & 0.97 \\
4 & 270.6 & 3.27 & 0.82 & 234.3 & 3.81 & 0.95 \\
8 & 150.8 & 5.87 & 0.73 & 127.0 & 7.03 & 0.88 \\
12 & 104.4 & 8.48 & 0.70 & 90.2 & 9.92 & 0.83 \\
\hline
\end{tabular}

and compares the two strategies of data distribution. The computations were performed on a Virgo cluster of computers, but in this case a Gigabit Ethernet network was used. The code was compiled with the full optimization option $O 3$ in order to make the ratio between the computation and communication speeds more challenging. In order to get more realistic estimates of the speedup and the efficiency coefficients, the initialization time $T_{\text {init }}$ was excluded from the computation time $T_{p}$, since this time can be neglected for real simulations. During the computational experiments, the solution was computed only for six time steps.

4.2. Parallel preconditioners. There have been many studies of the use of various ordering techniques to overcome the trade-off between parallelism and convergence in ILU factorization. Some new multicolor orderings were proposed by D'Azevedo et al. [2], Doi and Washio [11], Monga-Made and Van der Vorst [29], Čiegis [9]. The comparison of parallel preconditioners for nonsymmetric sparse linear systems was made by Ma [28].

A simple parallel version of the ILU preconditioner was implemented, with each processor computing the required factorization by using only a local part of the matrix $A$. Such a Jacobi type ILU preconditioner is fully parallel, but the convergence rate of the obtained iterations is decreased, (see also $[3,9]$ ). It is very difficult to estimate the convergence rate of the BiCGSTAB algorithm with the Jacobi ILU preconditoner even for problems obtained after discretization of the Laplace equation on uniform grids. The efficiency of preconditioners also depends strongly on the given problem coefficients and the properties of the grid. Therefore 
mainly experimental investigations are used for the analysis of simplified preconditioners. In Table 4.4, the performance of the BiCGSTAB iterative algorithm with the Jacobi ILU

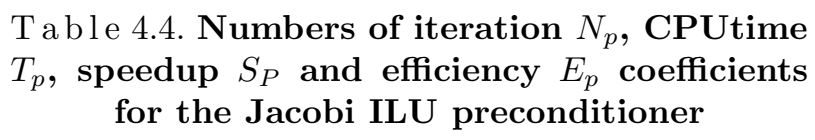

\begin{tabular}{|l|c|c|c|c|}
\hline$p$ & $N_{p}$ & $T_{p}$ & $S_{p}$ & $E_{p}$ \\
\hline 1 & 3304 & 1246 & 1.00 & 1.000 \\
2 & 3741 & 742 & 1.68 & 0.840 \\
4 & 4070 & 465.5 & 2.68 & 0.670 \\
8 & 4137 & 248.6 & 5.01 & 0.627 \\
12 & 4181 & 175.6 & 7.10 & 0.591 \\
\hline
\end{tabular}

preconditioner is given. Here, $N_{p}$ is the total number of the BiCGSTAB iterations calculated in solving all systems of linear equations by using $p$ processors, and $S_{p}$ and $E_{p}$ are the speedup and efficiency coefficients, respectively, of the parallel SuFiS algorithm. Note that the number of iterations was exactly the same for any number of processors in the previous experiments, i.e., the iterative process was always stopped after computing the maximal number of iterations. The computations were done on the ITWM Virgo cluster.

It can be seen that the number of iterations for the BiCGSTAB algorithm with the Jacobi ILU preconditioner increased as compared to the global ILU preconditioner. Therefore, the efficiency of parallel SuFiS is decreased (compare the new values of $E_{p}$ to the values given in Table 4.1). However, the quality of the Jacobi ILU preconditioner is still quite satisfactory.

The calculations of the ILU factorization and the solution of the problems $D x=f$ are quite costly. The efficiency of the sequential ILU preconditioner was compared the Jacobi diagonal preconditioner. The same problem was solved by using $N=9849$ iterations and $T_{1}=2315 \mathrm{CPU}$ time. Thus, the number of iterations increased 2.98 times, but the CPU time increased by only 1.85 times.

\section{Conclusions}

A parallel algorithm for the solution of mathematical models describing the filtering of solid particles from liquid oil in complicated 3D geometries is described. The governing NavierStokes-Brinkmann equations are discretized by the finite volume method, taking special care for accurate discretization of the velocity and the pressure on the interface between the pure fluid regions and the porous media region. The parallelization is based on the data decomposition method. The data are distributed among the processors by using two approaches. In the first approach, a structured reference grid is distributed using the optimal decomposition topology. In the second one, the general mesh is decomposed using the Metis library. A theoretical model is proposed for the estimation of the complexity of the given parallel algorithm. The theoretical and experimental results obtained are in very good agreement, and thus it can be predicted that the proposed parallel algorithm scales well, and it can be used efficiently for the simulation of oil filters with complicated 3D geometries.

Acknowledgements. Part of this work was supported by the EC under the project INTAS-30-50-4395, by the Kaiserslautern Excellence Cluster Dependable Adaptive Systems and Mathematical Modelling, DASMOD, and by Eureka Project EUREKA E!3691 OPTCABLES. 


\section{References}

1. Ph. Angot, Analysis of singular perturbations on the Brinkman problem for fictitious domain models of viscous flows. Math. Meth. Appl. Sci., 22 (1999), pp. 1395-1412.

2. E. F. D'Azevedo, P. A. Forsyth, and W. Tang. Ordering methods for preconditioned conjugate gradient methods applied to unstructured grid problems. SIAM J. Matrix Anal. Appl., 10 (1992), no. 4, pp. 567584 .

3. S. Balay, K. Buschelman, V. Eijkhout, W. D. Gropp, D. Kaushik, M. G. Knepley, L. Curfman McInnes, B. F. Smith, and H. Zhang, PETSc Users Manual. ANL-95/11 - Revision 2.3.0, Argonne National Laboratory, 2005.

4. R. Barrett, M. Berry, T. F. Chan, J. Demmel, J. Donato, J. Dongarra, V. Eijkhout, R. Pozo, C. Romine, H. Van der Vorst, Templates for the Solution of Linear Systems: Building Blocks for Iterative Methods. SIAM, Philadelphia, PA, 1994.

5. P. Bastian, Numerical Computation of Multiphase Flows in Porous Media. Habilitation Dissertation, Kiel university, 1999.

6. J. Bear, Y. Bachmat, Introduction to Modeling of Transport Phenomena in Porous Media. Kluwer Academic Publishers, Dordrecht etc., 1990.

7. A. Bruaset and A. Tveito, Numerical Solution of Partial Differential Equations on Parallel Computers. In: Lecture notes in computer science and engineering. Vol. 51, Springer, 2006.

8. A. Churbanov, A.Pavlov, and P. Vabishchevich, Operator-splitting methods for the incompressibel Navier-Stokes equations on non-staggered grids. I: First-order schemes. Int. J. Numer. Methods Fluids 21 (1995), no. 8, pp. 617-640.

9. R. Ciegis. Analysis of Parallel Preconditioned Conjugate Gradient Algorithms. Informatica, 16 (2005), no. 3, pp. 317-332.

10. R. Ciegis and V.],Starikovičius. Realistic performance tool for the parallel block LU factorization algorithm. Informatica, 14 (2003), no. 2, pp. 167-180.

11. S. Doi and T. Washio, Ordering strategies and related techniques to overcome the trade-off between parallelism and convergence in incomplete factorizations. Parallel Computing, 25 (1999), pp. 1995-2014.

12. J. H. Ferziger and M. Peric, Computational methods for fluid dynamics. Springer, 1999.

13. C. A. J. Fletcher, Computational techniques for fluid dynamics. Springer, Berlin etc., 1991.

14. P. Gresho and R. Sani, Incompressible flow and the finite element method. Advection-diffusion and isothermal laminar flow. In collaboration with M. S. Engelman. Chichester: Wiley, 1988.

15. Fraunhofer Institute for Industrial Mathematics, Annual Report, 2002. http://www.itwm.fhg.de/en/ zentral-berichte/annualreport2002/

16. Fraunhofer Institute for Industrial Mathematics, Department Flows and Complex Structures, http:// www.itwm.fhg.de/en/sks/indexsks/

17. A. Gupta, V.Kumar, and A. Sameh, Performance and scalability of preconditioned conjugate gradient methods on parallel computers. IEEE Transactions on Parallel and Distributed Systems, 6 (1997), no. 5, pp. $455-469$.

18. R. Hockney, Performance parameters and benchmarking on supercomputers. Parallel Computing, 17 (1991), pp. 1111-1130.

19. O. Iliev and V.Laptev, On numerical simulation of flow through oil filters. Comput. Vis. Sci., 6 (2004), pp. 139-146.

20. O. Iliev, V. Laptev, and D. Vassileva, Algorithms and software for computer simulation of flow through oil filters. Proc. FILTECH Europa, 2003, Düsseldorf, pp.327-334.

21. O. Iliev and D. Stoyanov, On a multigrid, local refinement solver for incompressible Navier - Stokes equations, Mathematical Modelling, 13 (2001), no. 8.

22. O. Iliev and D. Stoyanov, Multigrid adaptive local refinement solver for incompressible flows, Fraunhofer ITWM report No. 54, 2003.

23. G. Karypis and V. Kumar, Parallel multilevel $k$-way partitioning scheme for irregular graphs. SIAM Review, 41 (1999), no. 2, pp. 278-300.

24. M. Kaviany, Principles of Heat Transfer in Porous Media. Springer, New York etc., 1991.

25. V.Kumar, A. Grama, A. Gupta, and G. Karypis, Introduction to parallel computing: design and analysis of algorithms. Benjamin/Cummings, Redwood City, 1994.

26. H. P. Langtangen, Computational Partial Differential Equations. Numerical Methods and Diffpack Programming. Springer, Berlin, 2002. 
27. V. Laptev, Numerical solution of coupled flow in plain and porous media. PhD thesis, Technical University of Kaiserslautern, 2003.

28. S. Ma, Comparisons of the parallel preconditioners for large nonsymmetric sparse linear systems on a parallel computer. International Journal of High Speed Computing, 12 (2004), no. 1, pp. 55-68.

29. M. Monga-Made, H. A. Van der Vorst, A generalized domain decomposition paradigm for parallel incomplete LU factorization preconditionings. Future Generation Computer Systems, 17 (2001), pp. 925932.

30. P. Vabishchevich, The method of fictitious domains in problems of mathematical physics. (Metod fiktivnykh oblastej v zadachakh matematicheskoj fiziki.), Moskva: Izdatel'stvo Moskovskogo Univ., 1991.

31. A. A. Samarskii and P. N. Vabischevich, Computional Heat Transfer, Vol.1-02, John Wiley \& Sons Inc., New York, 1995.

32. S. Turek, Efficient solvers for incompressible flow problems. An algorithmic and computational approach. Lecture Notes in Computational Science and Engineering. 6. Berlin: Springer, 1999. 